\title{
Study of spallation by sub-picosecond laser driven shocks in metals
}

\author{
J.-P. Cuq-Lelandais ${ }^{1,2, a}$, M. Boustie ${ }^{1}$, L. Soulard ${ }^{2}$, L. Berthe ${ }^{3}$, T. De Rességuier ${ }^{1}$, P. Combis ${ }^{2}$, \\ J. Bontaz-Carion ${ }^{2}$, and E. Lescoute ${ }^{1}$ \\ 1 Institut P', ENSMA - CNRS - Université de Poitiers, UPR 3346, 1 avenue Clément Ader, \\ Téléport 3, 86961 Futuroscope Cedex, France \\ 2 CEA, DAM, DIF, 91297 Arpajon, France \\ 3 PIMM, Arts et Metiers ParisTech, 141 Boulevard de l'Hôpital, 75013 Paris, France
}

\begin{abstract}
Spallation induced by a laser driven shock has been studied for two decades on time scales of nanosecond order. The evolution of laser technologies now provides access to sources whose pulse duration is under the picosecond, corresponding to characteristic times of numerous microscopic phenomena. In this ultra-short irradiation regime, spallation experiments have been performed with time-resolved measurements of the free surface. In this solicitation type, damage occurs at small scale, leading to micrometric spalls. The VISAR measurements have been complemented with post-test observations and microtomography and compared with numerical simulations to check the models consistency of the laser-matter interaction, shock wave propagation and the dynamic damage criteria ability to reproduce spallation at this ultra-short time scale, inducing strong tensile stress states at very high strain rates.
\end{abstract}

\section{Introduction}

Dynamic damage characterization of materials presents a great interest for industry fields as aeronautic, automobile, but also research applications like geology. One way to investigate it is the generation of high pressure shock waves. Actually, several methods exist, as plate impact or explosives. However, such processes are generally destructive and the strain rates expected are limited $\left(10^{5} \mathrm{~s}^{-1}\right.$ maximum) [1]. This can be avoided by using high energy laser short pulses to generate a pressure wave. The characteristic durations involved are mainly about the nanosecond range and strain rates about $10^{7} \mathrm{~s}^{-1}$. The latest laser technologies evolutions provide an access to shorter regimes in durations, going below the picosecond. This kind of regime is leading to extreme dynamic solicitations in the target, and spallation at a micrometric scale [2].

In this work, spallation by laser driven shock experiments have been performed on the femtosecond LULI's 's "100TW" facility in 2008 , with a laser beam of $1.06 \mu$ m wavelength, up to $30 \mathrm{~J}$ energy and a Gaussian pulse of $300 \mathrm{fs}$ full width at half maximum. The laser is focused on a $100 \mu \mathrm{m}$ thick tantalum sample, with a $2 \mathrm{~mm}$ impact diameter. In this configuration, we can obtain laser intensities up to $3 \mathrm{PW} / \mathrm{cm}^{2}\left(1 \mathrm{PW}=10^{15} \mathrm{~W}\right)$. As a time resolved diagnosis, a VISAR measurement of free surface velocity is used [3]. The data obtained can inform about the shock propagation and damage history by comparing it with numerical results computed by hydrocodes. In this case, a good numerical

\footnotetext{
a e-mail: jean-paul.cuq-lelandais@lcd.ensma. fr

${ }^{b}$ LULI = Laboratoire d'Utilisation des Laser Intenses - UMR CNRS 7605 (Ecole Polytechnique, France) Website: http//www.luli-polytechnique.fr
}

This is an Open Access article distributed under the terms of the Creative Commons Attribution-Noncommercial License 3.0, which permits unrestricted use, distribution, and reproduction in any noncommercial medium, provided the original work is properly cited. 


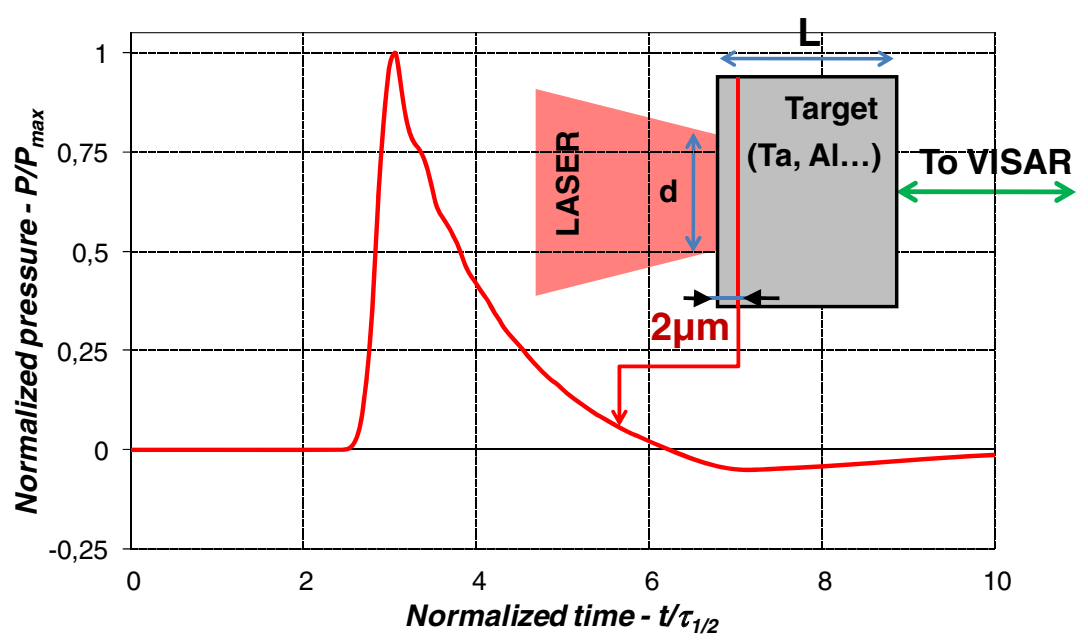

Fig. 1. Calculated pressure loading $2 \mu \mathrm{m}$ depth equivalent to a LULI $100 \mathrm{TW}$ irradiation.

free surface velocity restitution is the key to validate one dynamic damage model and determine its parameters.

In the following paragraphs, we detail the procedure used to obtain parameter set for the Kanel empirical ductile damage model [4] at high strain rate. The numerical study is divided into three steps: first, the pressure loading characterization driven by the laser interaction with the solid medium. Then, the shock wave propagation will be discussed and finally the damage model will be dimensioned to fit experimental data. In addition, the parameters in femtosecond regime are compared to the values obtained with other spall generators (plate impact, nanosecond laser) to see the evolution with strain rate.

\section{Laser-matter interaction in ultrashort regime}

The first step in this work is to characterize with accuracy the pressure loading history on the laser irradiated face. The equivalent time solicitation is linked to the laser-matter interaction in fs regime. The pressure generation in this ultra-short regime is very different from nanosecond laser regimes where the compression is induced only by action-reaction with the expanding plasma. In femtosecond regime, the plasma has not enough time to expand and the laser interacts only with the solid by this way:

The incident ultra-short beam reaches the target and initially excites the electrons on the front face, creating an electron-ion non-equilibrium state [5]. The absorbed energy is then transmitted to the crystal lattice via collisions between particles, which causes a strong gradient in temperature on a thin thickness of the front face target, about a micron deep. The relaxation time of this imbalance is about ten picoseconds, which is considerable in comparison of the pulse duration [6]. The gradient of temperature imposed causes phases transitions at high-speed rates, vaporizing and melting the matter which is ejected, leading to an ablation of matter in the micron range [7]. This yields to a pressure profile at the origin of a shock wave which propagates in-depth within material.

This equivalent pressure profile is calculated using the laser-matter interaction code ESTHER provided by the CEA-DAM of Bruyères-le-Châtel [8]. This code includes particularly a two temperatures model (2TM) describing the electron-ion non-equilibrium state.

Figure 1 represents the equivalent normalized pressure profile induced by a 300 fs FWHM Gaussian laser in the target at a $2 \mu \mathrm{m}$ depth. Several simulations at different laser intensities showed the solicitation shape and its middle height duration does not vary for one given material. Thus, it can be characterized by its maximum pressure, given by a power law and its middle height duration, constant for one material. The intensity/maximum pressure values sets and the FWHM profile/laser durations 
New Models and Hydrocodes for Shock Wave Processes in Condensed Matter

Table 1. Equivalent pressure profile data for aluminium \& tantalum submitted to a LULI100TW laser.

\begin{tabular}{|c|c|c|c|c|c|}
\hline \multirow{2}{*}{ Material } & \multirow{2}{*}{$\begin{array}{l}\text { Pressure history } \\
\text { Depth }(\mu \mathrm{m})\end{array}$} & \multirow{2}{*}{$\begin{array}{c}\text { Normalized profile } \\
\text { FWHM duration }\left(\tau_{1 / 2} / \tau\right)\end{array}$} & \multicolumn{2}{|c|}{$\begin{array}{c}\text { Maximum Pressure } \\
P_{\max }^{2 \mu m}=A \cdot \Phi^{n} \\
\end{array}$} & \multirow{2}{*}{$\begin{array}{c}\text { Intensity } \\
\text { validity } \\
\text { interval } \\
\left(\mathrm{PW} / \mathrm{cm}^{2}\right) \\
\end{array}$} \\
\hline & & & $\mathrm{A}$ & $\mathrm{n}$ & \\
\hline Aluminium & $2 \mu \mathrm{m}$ & 230 & 1.70 & 0.74 & {$\left[10^{13} ; 5 \cdot 10^{15}\right]$} \\
\hline Tantalum & $2 \mu \mathrm{m}$ & 350 & 2.18 & 0.52 & {$\left[10^{13} ; 5 \cdot 10^{15}\right]$} \\
\hline
\end{tabular}

${ }^{\mathrm{c}}$ The table coefficients are compatibles with pressures in $\mathrm{M}$ bar order and intensities in $\mathrm{PW} / \mathrm{cm}^{2}$.
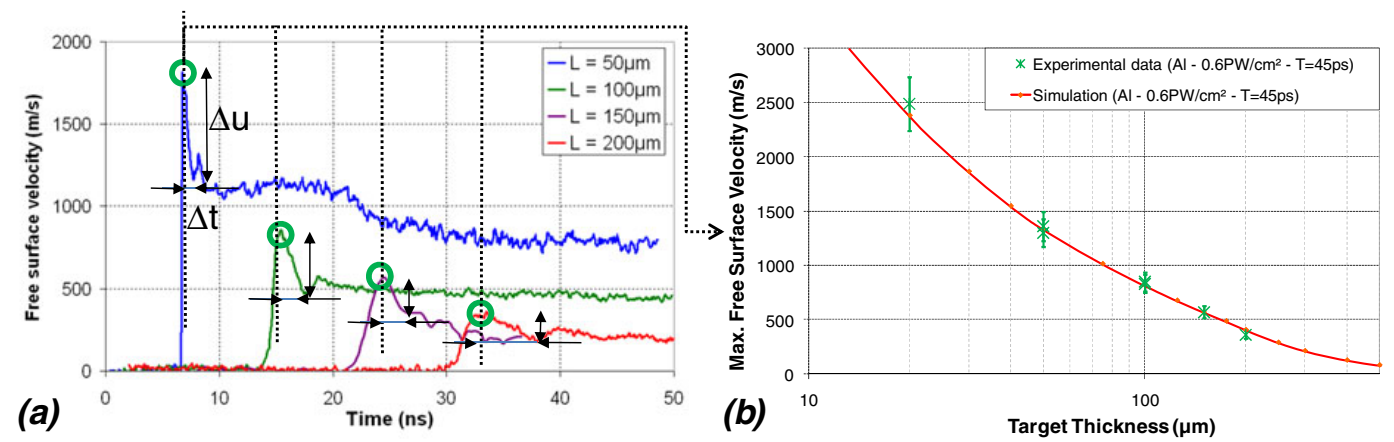

Fig. 2. Experimental free surface velocities of laser shocked aluminium targets of different thicnesses at the same intensity (2a); Experimental/Numerical comparison of the maximum free surface velocities withe target thickness at a $0.6 \mathrm{PW} / \mathrm{cm}^{2}$ laser intensity $(2 \mathrm{~b})$.

ratios are reported for aluminium and tantalum subjected to a $300 \mathrm{fs}$ laser with a $1.06 \mu \mathrm{m}$ wavelength in the Table 1 . In each case, we can notice that a $300 \mathrm{fs}$ laser pulse results into a pressure profile whose characteristic duration is roughly two decades greater at width medium height. This enlargement has also been observed in ns regime, but not in such a scale [9].

\section{Shock wave propagation \& spallation simulation}

\subsection{Shock wave propagation and decay}

In order to check the ability of numerical codes to simulate shock wave propagation in this ultra-short regime, numerical simulations have been performed with the one-dimensional hydrodynamic code called SHYLAC. Initially, we performed a simulation with an elasto-plastic behavior without damage criteria. Because the pressure load calculated by ESTHER is short and intense, inducing a strong hydrodynamic attenuation [10], it is important to check the relevance of the code to model properly this decay. To achieve this, the experimental speed jump at shock breakout on the free surface velocity is compared to the numerical model. Figure 2(a) shows several examples of VISAR measurements for $100 \mathrm{TW}$ shots at the same intensity on aluminium targets of increasing thicknesses. The maximum velocities are reported on Figure 2(b) and superposed on the numerical equivalents shock velocities plotted in function of target thickness. The comparison provides a good agreement between simulation and experiments, validating the shock propagation modelling, and also the mechanical loading calculated with ESTHER.

\subsection{Damage modelling}

We then integrated into the numerical model an active damage criterion in order to represent the spallation induced by the tension state. We chose the model of Kanel, based on the growth of a 


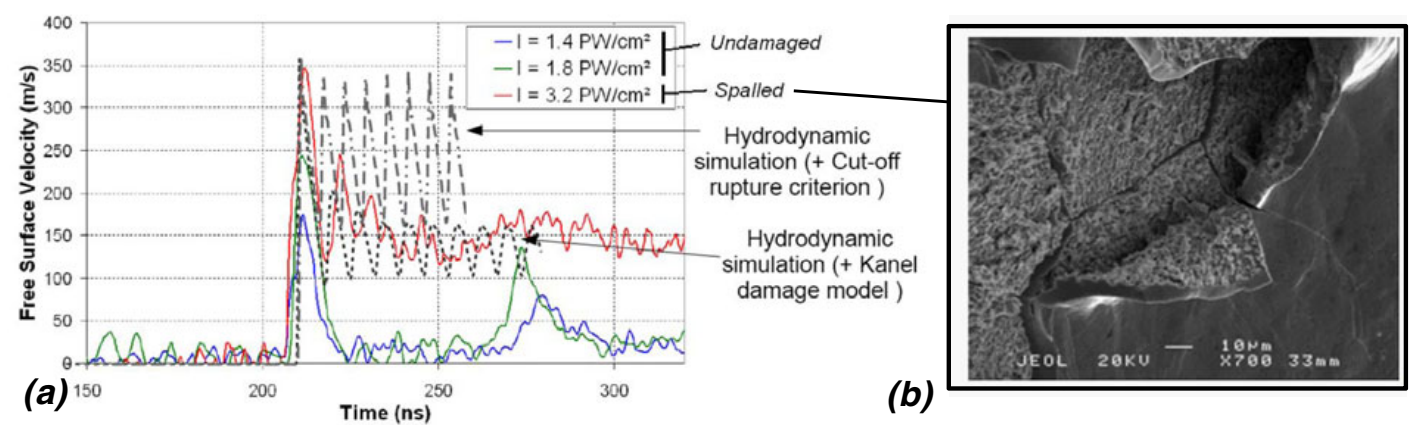

Fig. 3. Experimental free surface velocities of laser shocked $100 \mu \mathrm{m}$ tantalum targets for different intensities Numerical Kanel \& Cut-off fitting (3a) ; SEM picture of the damaged free surface corresponding to the highest intensity on the $3 \mathrm{a}$ graph (3b).

void volume starting from a given threshold in tension and described by the following differential equation (1) [4].

$$
\begin{cases}\frac{d V_{t}}{d t}=-k \cdot \operatorname{sign}(P) \cdot\left(|\sigma|-\sigma_{d}\right)\left(V_{t}+V_{t 1}\right) & \text { if }|\sigma| \geq \sigma_{d} \\ \frac{d V_{t}}{d t}=0 & \text { if }|\sigma|<\sigma_{d}\end{cases}
$$

Where $V_{t}$ is the void rate, $\sigma$ ghe axial stress, $\sigma_{d}$ the threshold stress and $P$ the pressure. $k$ and $V_{t 1}$ are characteristic of the material. The evolution of the void rate retroacts on the material mechanical properties (equations (2) to (4)):

$$
\begin{gathered}
\sigma_{s}=\sigma_{s}^{0} \cdot \frac{V_{t 1}}{V_{t 1}+V_{t}} \\
G=G^{0} \cdot \frac{V_{t 1}}{V_{t 1}+V_{t}} \\
Y=Y^{0} \cdot \frac{V_{t 1}}{V_{t 1}+V_{t}}
\end{gathered}
$$

Where $\sigma_{d}^{\mathrm{o}}, \mathrm{G}^{\mathrm{o}}, \mathrm{Y}^{\mathrm{o}}$ are respectively the initial threshold stress, shear modulus and yield strength. One of the main advantages of this model is the number of parameter to find, only three. This makes possible to determine them by fitting the experimental VISAR signal. Figure 3(a) shows an example of Kanel fitting for a $100 \mu \mathrm{m}$ tantalum target subjected to the $100 \mathrm{TW}$ with different intensities. For the two lowest cases, which are undamaged, the velocity come back to zero after the shock wave break out and come back again later after have travelled back and forth in the target. Only the highest intensity makes the target spall, visible by the final ejection $150 \mathrm{~m} / \mathrm{s}$ velocity. Post-shock damaged rear surface SEM observation confirms it (Fig. 3(b)). This latter allows to see the fracture aspect which typical of transgranular ductile damage. In the other cases, the velocity come back to zero after the shock wave break out and come back again later after have travelled back and forth in the target.

For the spalled case, two simulations with damage have been fitted. First we have used a cut-off criterion $\left(\sigma_{R}=80 \mathrm{GPa}\right)$, but the curve does not match with the experimental signal: the average final spall ejection velocity is too high and oscillations too enhanced. This is certainly due to the simplicity of this criterion which does not represent correctly the dynamic aspects of the spall damage. Results are better with the Kanel model, more physical in the description. The ejection velocity is well reproduced. The corresponding data set is reported in theTable 2. 
New Models and Hydrocodes for Shock Wave Processes in Condensed Matter

Table 2. Tantalum Kanel damage parameters fitted for different shock generators/strain rates.

\begin{tabular}{|c|c|c|c|c|c|}
\hline Shock generator & Tensionduration $(\mathbf{n s})$ & Strain rate $\left.\mathbf{( s}^{-1}\right)$ & $\sigma_{R}$ (kbar) & K & Vt1 \\
\hline Plate impacts $[14]$ & 1500 & $10^{5}$ & 45 & 0.0079 & 0.00058 \\
\hline LCD laser & 30 & $2 \cdot 10^{6}$ & 69 & 0.026 & 0.0019 \\
\hline LULI100TW & $\mathbf{0 . 5}$ & $\mathbf{2} \cdot \mathbf{1 0}^{7}$ & $\mathbf{8 1}$ & $\mathbf{0 . 2 8}$ & $\mathbf{0 . 0 2}$ \\
\hline Evolution & $\boldsymbol{\nearrow}$ & $\boldsymbol{\lambda}$ & $\boldsymbol{\nearrow}$ & $\boldsymbol{\nearrow}$ & $\boldsymbol{\nearrow}$ \\
\hline
\end{tabular}

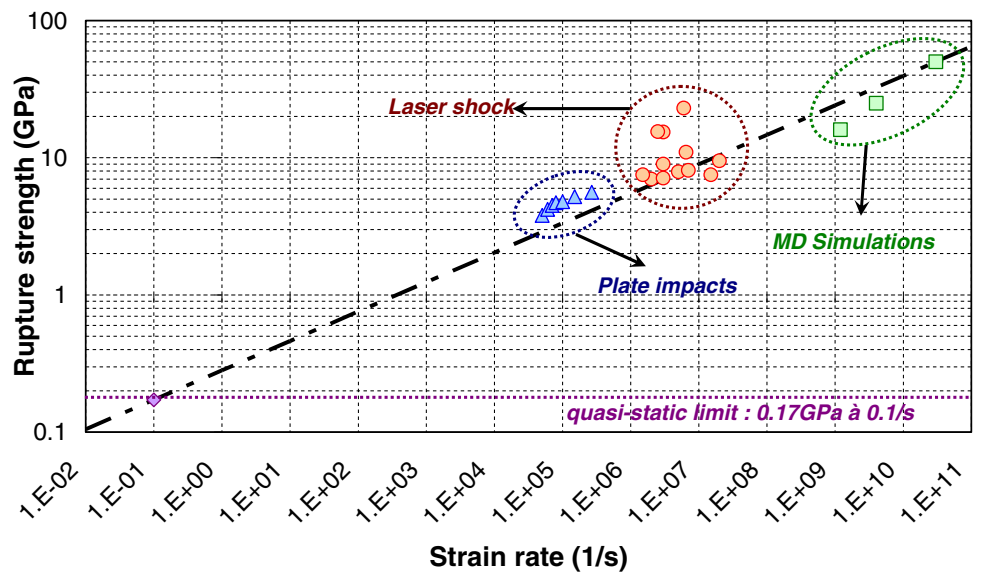

Fig. 4. Failure stress / strain rate diagram for tantalum obtained with plate impacts [14], laser driven shock and MD simulations.

\section{Damage evolution with strain rate}

Damage and rupture conditions, as many other mechanical related phenomena (elasticity, plasticity ... etc), are strain rate dependants [11]. This fact is experimentally illustrated in femtosecond regime with the experimental data on Fig. 2(a): from one VISAR signal, it is possible to extract information about spallation strength and strain rate by measuring respectively the velocity pullback amplitude $\Delta \mathrm{u}$ and duration $\Delta \mathrm{t}$ (See Equations (5) and (6)) [12].

$$
\sigma_{\text {spall }}=\frac{1}{2} \cdot \rho_{o .} c_{o} \cdot \Delta u
$$

In the Fig. 2(a), when the target thickness is reduced, spall strength and strain rate are both increased. This opens the possibility to study damage mechanics at very high strain rates $\left(10^{8} \mathrm{~s}^{-1}\right.$ in fs regime). The strain rate comparison can be achieved by using transversally several shock generators. Figure 4 shows the rupture strength in tension obtained by different setups for tantalum. When the strain rate is increased, the failure stress raises with a power law type. At ultra high strain rate, the added results obtained by Molecular Dynamics (MD) simulations of shock are in continuity with all experimental points [13].

$$
\dot{\varepsilon}=\frac{\Delta u}{\Delta t} \cdot \frac{1}{2 \cdot c_{o}}
$$

This increased resistance to failure with strain rate can be transposed to the Kanel damage model. This latter does not take in account the strain rate effects in its formulation. We have thus compared the evolution when strain rate changes significantly. The Kanel parameters have been fitted by using VISAR spalled signals coming from longer shockwaves generators (nanosecond laser and plate impacts). All the results are given in the Table 2 and highlight a tendency. As expected, the stress threshold $\sigma_{R}$ increases with strain rate, as all the other parameters $\mathrm{K}$ and $\mathrm{Vt} 1$. 


\section{Conclusion \& future work}

Experiments on tantalum and aluminium targets showed the possibility to use spallation data to investigate the dynamic damage field at very high strain rate. The comparison between experimental free surface velocities evidences that fs irradiation regime is in the continuity of shocks physics into this extremely short duration scales, from shock propagation to spallation. It also validated the use of the laser-matter modelisation with a two-temperature model describing the electron-ion non-equilibrium state, non negligible at the sub-picosecond scale.

We shown that Kanel damage model was able to reproduce well the ductile rupture of tantalum. The strain rate model dependence has been highlighted by fitting all its parameters with experimental results from several shock generators which have different strain rates domains.

One other interesting result is that MD spallation simulations at the sub-micrometric scale are able to predict failure strengths at ultra high strain rates $\left(\sim 10^{10} \mathrm{~s}^{-1}\right)$ which are consistent with the experimental behaviour continuity. Based on these encouraging results, it is planned to use MD simulation to determine Kanel parameters by using the simulated free surface velocity. This work could be associated with microscopic study of damage by spallation in order to understand micro-mechanisms responsible of ductile damage at these extreme solicitations.

We are very grateful to all the LULI staff who helped in building these experiments and trained us to run the 100TW laser facility: P. Audebert, J. Albrecht, J. Fuchs, C. Le Bris, Ji Ping Zou, S. Baton.... Many thanks to CEA researchers from CEA for joining this work, and particularly for the numerical part with "CEA/ESTHER" code kindly provided by CEA to our labs.

\section{References}

1. R. Kinslow, High velocity impact phenomena (Academic Press, New-York/London, 1971).

2. H. Tamura, T. Kohama, K. Kondo et al, J. Appl. Phys., 89 3520, (2001).

3. L.M. Barker and R.E. Hollenback, J. Appl. Phys., 434669 (1972).

4. G.I. Kanel and V.E. Fortov, Adv. Mech., 10 3, (1987).

5. B. Rethfeld, A. Kaiser, M. Vikanek and G. Simons, Phys. Rev. B, 65 21403, (2002).

6. H.E Elsayed-Ali, T.B. Norris, M.A Pessot and G.A. Mourou, Phys. Rev. Lett., 68 2834, (1992).

7. S. Bruneau, J. Hermann, T. Itina et al, J. Phys. IV France, 108 41, (2003).

8. J.-P. Colombier, P. Combis, R. Stoian and E. Audouard, Phys. Rev. B 75 104105, (2007).

9. S. Couturier, T. De Rességuier, M. Hallouin et al, J. Appl. Phys. 79 9338, (1996).

10. E. Moshe, S. Eliezer, E. Dekel et al, J. Appl. Phys., 83 4004, (1998).

11. J.-P. Cuq-Lelandais, M. Boustie, L. Berthe et al, J. Phys D, 42 065402, (2009).

12. T. Antoun, L. Seaman, D.R. Curran et al, Spall fracture, (Springer-Verlag, New-York, 2002).

13. L. Soulard, This conference, (2010).

14. G. Roy, Vers une modélisation approfondie de l'endommagement ductile dynamique. Inverstigation expérimentale d'une nuance de tantale et développements théoriques (Thèse de l'université de Poitiers, 2003). 